\title{
Effect of thermo diffusion on mixed convective heat and mass transfer flow of micropolar fluid in a circular annulus with heat sources
}

\author{
T. Suvarna ${ }^{1}$ and Dr. M. Ravindra ${ }^{2}$ \\ ${ }^{1}$ Research Scholar, Department of Mathematics ,Rayalaseema University, Kurnool, A.P,Iindia \\ ${ }^{2}$ Department of Mathematics, S.S.B.N. Degree \& PG College(Autonomous), Anantapuramu,A.P,India
}

\section{Abstract :}

In this paper, we make an attempt to investigate combined influence of magnetic field and dissipation on connective heat and mass transfer flow of a viscous chemically reacting fluid through a porous medium in the concentric cylindrical annulus with inner cylinder maintained at constant temperature and concentration on the other cylinder maintained constant heat flux. The equations governing the flow, heat. Mass and micro rotation are solved by employing Galerkin finite element analysis with quadratic approximation functions. The temperature, concentration and micro concentration distributions are analyzed for different values of $\mathrm{Sr}, \gamma, \mathrm{Ec}, \Delta, \lambda$ and $\mathrm{A}$. The rate of heat and mass transfer and couple stress are numerically evaluated for different variations of the governing parameters.

Keywords : Soret effect, heat sources, heat and mass transfer, Circular annulus, micropolar fluid.

\section{INTRODUCTION:}

The theory of micropolar fluids initiated by Erigen [3] exhibits some microscopic effects arising from the local structure and micro motion of the fluid elements. Further, they can sustain couple stress and include classical Newtonian fluid as a special case. The model of micropolar fluid represents fluids consisting of rigid randomly oriented (or sphenical) particles suspended in a viscous medium where the deformation of the particles is ignored. The fluid containing certain additives, some polymeric fluids and animal blood are examples of micropolar fluids. The mathematical theory of equations of micropolar fluids and application of these fluids in the theory of lubrication and porous media is presented by Lukaszewics [7]. Agarwal and Dhanpal [1] obtained numerical solution of micropolar fluid flow and heat transfer between two co-axial porous circular cylinders. Verma and Singh [16] have analyzed the behaviour of parametric fluid flow in a porous annulus in the presence of external magnetic field acting parallel to the common axis of the long co axial porous cylindrical tubes. Panja et al. [11] studied the flow of electrically conducting Reiner - Rivlin fluid between two non-conducting co axial circular cylinders with porous walls in the presence of uniform magnetic field. Shivashankar et al. [12] have obtained numerical solution to the MHD flow of micropolar fluid between two concentric porous cylinders; Murthy et al. [9] have considered study flow of micropolar fluid through a circular pipe and transverse with constant suction and injection.

An enclosed cylindrical annular cavity formed by three vertical, concentric cylinders, containing a fluid through which heat is transferred by natural convection, is a simplified representation of a number of practical and experimental situations. Also, the annulus represents a common geometry employed in a variety of heat transfer systems ranging from simple heat exchangers to the most complicated nuclear reactors. Since, the flow and heat transfer in a cylindrical annular configuration contains all the essential physics that are common to all confined 
natural convective flows, a complete understanding of the flow in such geometry is very essential. In addition, from a computational stand point, the annular configuration allows investigation of a wide range of geometrical effects. Convection is an important phenomenon in the crystal growth techniques as it can account for heat transfer in the liquid phase and can change the material properties.

Gebhart and Mollendorf [6] have shown that viscous dissipation heat in the natural convective flow is important when the flow field is of extreme size at extremely low temperature or in high gravitational field. On the other hand Barletta [2] have pointed out that relevant effects of viscous dissipation on the temperature profiles and on Nusselt number my occur in the fully developed forced convection in tubes. In view of this several authors notably Soundalgekar and Pop [15]. Shivasankar et al. [13], Sreevani [14] and Barletta [2] have studied the effect of viscous dissipation on the convective flows past an infinite vertical plate and through vertical channels and ducts. The effect of viscous dissipation on natural convection has been studied for some different cases including the natural convection from horizontal cylinder embedded in a porous media by Fand and Brucker [7], Giampietrao Fabbri [5] and Sreevani [14]. They reported that the viscous dissipation may not be neglected in all cases of natural convection from horizontal cylinders and further that the inclusion of a viscous dissipation term in a porous medium may lead to more accurate correlation equations, the effect of viscous dissipation has been studied by Nakayama and Pop [10] for steady free convection boundary layer over a non-isothermal body of arbitrary shape embedded in porous media. They used the integral method to show that the viscous dissipation results in lowering the level of the heat transfer rate from the body. This observation has been pointed also by Murthy and Singh [8] for the natural connection flow along an isothermal wall embedded in a porous medium. They concluded that that the effect of viscous dissipation increases as we move from Non-Darcy regime to Darcy regime. Recently, Prasuna et al [17] have analysed the effect of dissipation and Soret effect on convective heat and mass transfer flow through a porous mediun in a concentric annulus.

\section{FORMULATION OF THE PROBLEM:}

We consider the steady flow of an incompressible, viscous, electrically conducting micropolar fluid through a porous medium in an annulus region between the concentric porous cylinders $r=a$ and $r=b(b>a)$ under the influence of a radial magnetic field $\frac{H_{0}}{r^{2}}$.

The fluid is injected through the inner cylinder with radial velocity $u_{a}$ and flows outward through the outer cylinder with a radial velocity $\mathrm{u}_{\mathrm{b}}$. We also take the viscous, Darcy and Ohmic dissipation into account

The velocity and micro rotation are taken in the form

$$
\left.\begin{array}{lll}
\mathrm{v}_{\mathrm{r}}=\mathrm{u}(\mathrm{r}), & \mathrm{v}_{\theta}=\mathrm{v}=0, & \mathrm{v}_{\mathrm{z}}=\mathrm{w}(\mathrm{r}) \\
\omega_{\mathrm{r}}=0, & \omega_{\theta}=\omega(\mathrm{r}), & \omega_{\mathrm{z}}=0
\end{array}\right\}
$$

The equations governing the flow and heat and mass transfer eqn.(1)

$$
\begin{aligned}
& \frac{\partial u}{\partial r}+\frac{u}{r}=0 \\
& \rho u \frac{\partial u}{\partial r}=-\frac{\partial \phi}{\partial r}+(\mu+k)\left(\frac{\partial^{2} u}{\partial r^{2}}+\frac{1}{r} \frac{\partial u}{\partial r}-\frac{u}{r^{2}}\right)-\left(\frac{\mu}{k_{1}}\right) u \\
& \rho u \frac{\partial w}{\partial r}=-\frac{\partial p}{\partial z}+(\mu+k)\left(\frac{\partial^{2} w}{\partial r^{2}}+\frac{1}{r} \frac{\partial w}{\partial r}\right)-\rho \bar{g}-\left(\frac{\mu}{k_{1}}\right) w-k \frac{\partial w}{\partial r}(r \omega)
\end{aligned}
$$




$$
\begin{gathered}
\rho j u \frac{\partial \omega}{\partial r}=r\left(\frac{\partial^{2} \omega}{\partial r^{2}}+\frac{1}{r} \frac{\partial \omega}{\partial r}-\frac{N}{r^{2}}\right)-k \frac{\partial w}{\partial r}-2 k \omega \\
\rho_{0} C_{p} w \frac{\partial T}{\partial z}=k_{f}\left(\frac{\partial^{2} T}{\partial r^{2}}+\frac{1}{r} \frac{\partial T}{\partial r}\right)+(2 \mu+k)\left[\left(\frac{\partial u}{\partial r}\right)^{2}+\frac{\mu^{2}}{r^{2}}+\frac{1}{2}\left(\frac{\partial w}{\partial r}\right)^{2}\right] \\
+2 k\left(\frac{1}{2} \frac{\partial w}{\partial r}+\omega\right)^{2}-2 \frac{\beta}{r} \omega \frac{\partial \omega}{\partial r}+r\left(\left(\frac{\partial \omega}{\partial r}\right)^{2}+\frac{\omega^{2}}{r^{2}}\right)-Q_{H}\left(T-T_{e}\right) \\
w \frac{\partial C}{\partial z}=D_{m}\left(\frac{\partial^{2} C}{\partial r^{2}}+\frac{1}{r} \frac{\partial C}{\partial r}\right)-\gamma^{\prime} C+\frac{D_{m} K_{T}}{T_{m}}\left(\frac{\partial^{2} T}{\partial r^{2}}+\frac{1}{r} \frac{\partial T}{\partial r}\right) \\
\rho-\rho_{o}=-\beta \rho_{o}\left(T-T_{o}\right)-\beta^{\bullet} \rho_{o}\left(C-C_{o}\right)
\end{gathered}
$$

where $\mathrm{u}, \mathrm{w}$ are the velocity components along $\mathrm{O}(\mathrm{r}, \mathrm{z})$ directions, $\mathrm{T}$ is the temperature, $\omega$ is the micro rotation, $p$ is the pressure, $\rho$ is the density, $\mu$ is the dynamic viscosity, $C_{p}$ is the specific heat at constant pressure, $\mathrm{k}_{\mathrm{f}}$ is the thermal conductivity, $\mathrm{k}_{1}$ is the permeability of the porous permeability, $\sigma$ is the electrical conductivity $\mu$ is the magnetic permeability and $\mathrm{k}, \mathrm{r}, \beta$ are the material constants.

The boundary conditions are

$$
\begin{aligned}
& \mathrm{u}=\mathrm{ub}, \quad \mathrm{w}=0, \quad \omega=0, \quad \mathrm{~T}=\mathrm{T}_{0}+\mathrm{A}_{0} \mathrm{z}, \mathrm{C}=\mathrm{C}_{0}+\mathrm{B}_{0} \mathrm{z} \quad \text { on } \quad \mathrm{r}=\mathrm{a} \\
& \mathrm{u}=\mathrm{ua}, \quad \mathrm{w}=0, \quad \omega=0, \quad \mathrm{~T}=\mathrm{T}_{1}+\mathrm{A}_{0} \mathrm{Z}, \mathrm{C}=\mathrm{C}_{1}+\mathrm{B}_{0} \mathrm{Z} \quad \text { on } \quad \mathrm{r}=\mathrm{b}
\end{aligned}
$$

From the equation of continuity we obtain

$$
r u=c, \text { constant } \Rightarrow r u=a u_{a}=b u_{b} \quad \Rightarrow u=\frac{a u_{a}}{r}
$$

In view of the boundary condition on temperature and concentration, we may write

$$
\mathrm{T}=\mathrm{T}_{0}+\mathrm{A}_{0}(\mathrm{z})+\theta(\mathrm{r}), \mathrm{C}=\mathrm{C}_{0}+\mathrm{B}_{0}(\mathrm{z})+\phi(\mathrm{r})
$$

On introducing the non-dimensional variables $\mathrm{r}^{\prime}, \mathrm{w}^{\prime}, \theta^{\prime}, \mathrm{p}^{\prime}$ and $\mathrm{N}^{\prime}$ as

$$
r^{\prime}=\frac{r}{a}, w^{\prime}=\frac{w}{v / a}, \theta=\frac{T-T_{0}}{T_{i}-T_{0}}, \omega^{\prime}=\frac{(\mu+k) \omega}{\rho\left(\mu^{2} / a^{2}\right)}, p^{\prime}=\frac{p}{\rho\left(\mu^{2} / a^{2}\right)}, \phi=\frac{C-C_{0}}{C_{i}-C_{0}}
$$

The equations (2) - (4) reduces to (on dropping the dashes)

$$
\begin{gathered}
w_{r r}+\left(1-\frac{S}{1+\Delta}\right) \frac{1}{r} w_{r}=-\pi_{1}+\frac{M^{2}}{r^{2}} w+D_{1}^{-1} w-G_{1}(\theta+N \phi)-\frac{\Delta_{1}}{r} \frac{\partial}{\partial r}(r \omega) \\
\omega_{r r}+(1-S A) \frac{1}{r} \omega_{r}-\left(\frac{1}{r^{2}}-\frac{2 \Delta}{A}\right) \omega=\frac{\Delta_{1}}{A r} \frac{d w}{d r} \\
P_{r} N_{T} w=\theta_{r r}+\frac{1}{r} \theta_{r}-\alpha \theta+E c P_{r}\left\{\begin{array}{c}
(2+\Delta)\left(\frac{S^{2}}{r^{4}}+w_{r}^{2}\right)+2 \Delta\left(\frac{1}{2} w_{r}+\omega\right)^{2} \\
-2 \Delta \omega \frac{\partial \omega}{\partial r}+A_{1}\left(w_{r}^{2}+\frac{\omega^{2}}{r^{4}}\right)
\end{array}\right\} \\
S c N_{c} w=\phi_{r r}+\frac{1}{r} \phi_{r}-\gamma \varphi+\operatorname{ScSo}\left(\theta_{r r}+\frac{1}{r} \theta_{r}\right)
\end{gathered}
$$

where 
$G=\frac{\beta g a^{3}\left(T_{i}-T_{0}\right)}{v^{2}}($ Grashof number $), D^{-1}=\frac{a^{2}}{k_{1}}$ (Darcy parameter $)$

$P_{r}=\frac{\mu C_{p}}{k_{f}}$ (Prandtl number), $\quad E c=\frac{a^{2}}{C_{p}\left(T_{i}-T_{0}\right) v^{2}} \quad$ (Eckert number)

$\alpha=\frac{Q_{H} a^{2}}{k_{f} C_{p}}$ (Heat source parameter), $S=\frac{a u_{a}}{v}$ (Suction parameter)

So $=\frac{D_{m} K_{T}\left(C_{i}-C_{0}\right)}{T_{m}\left(T_{i}-T_{0}\right)} \quad$ (Soret parameter), $\quad \gamma=\frac{\gamma^{\prime} a^{2}}{D_{m}} \quad$ (Chemical Reaction parameter),

$A=\frac{r}{\mu a^{2}} \quad \Delta=\frac{\mu}{k} \quad$ (Micropolar parameters)

$\Delta_{1}=\frac{\Delta}{1+\Delta}, \quad D_{1}^{-1}=\frac{D^{-1}}{1+\Delta}, \quad G_{1}=\frac{G}{1+\Delta}, \quad r=\frac{b}{a}$

The non-dimensional boundary conditions are

$$
\begin{array}{llllll}
\mathrm{w}=0, & \theta=1, & \phi=1 & \mathrm{~N}=0 & \text { on } & \mathrm{r}=1 \\
\mathrm{w}=0, & \theta=0, & \phi=0 & \mathrm{~N}=0 & \text { on } & \mathrm{r}=\mathrm{s}
\end{array}
$$

\section{METHOD OF SOLUTION:}

The finite element analysis with quadratic polynomial approximation functions is carried out along the radial distance across the circular cylindrical annulus. The behavior of the velocity, temperature and concentration profiles has been discussed computationally for different variations in governing parameters. The Gelarkin method has been adopted in the variation formulation in each element to obtain the global coupled matrices for the velocity, temperature and concentration in course of the finite element analysis. Choose an arbitrary element $e_{k}$ and let $w^{k}, \theta^{k}$ and $N^{k}$ be the values of $\mathrm{w}, \theta$ and $\mathrm{N}$ in the element $\mathrm{e}_{\mathrm{k}}$.

We define the error residuals as

$$
\begin{aligned}
& E_{w}^{k}=\frac{d}{d r}\left(r \frac{d w^{k}}{d r}\right)-\frac{\lambda}{1+\Delta} \frac{d w^{k}}{d r}+\Delta_{1}\left(r \omega^{k}\right)+ \\
& \pi_{1} r-D^{-1} r w^{k}-G r\left(\theta^{k}+N \varphi^{k}\right) \\
& E_{\theta}^{k}=\frac{d}{d r}\left(r \frac{d \theta^{k}}{d r}\right)-P_{r} N_{t} w_{r}-\alpha \theta+E c P_{r}\left\{\begin{array}{l}
\left.(2+\Delta) \frac{\lambda^{2}}{r^{3}}+((2+\Delta) r+A)\left(\frac{d w}{d r}\right)^{2}+\right) \\
\left.2 \Delta r\left(\frac{1}{2} \frac{d w}{d r}+\right)^{2} \omega-2 A \omega \frac{d \omega}{d r}+A_{1} \frac{\omega^{2}}{r}\right)
\end{array}\right\} \\
& E_{C}^{k}=\frac{d}{d r}\left(r \frac{d \phi^{k}}{d r}\right)-S c N_{C} w_{r}-\gamma \phi^{k}+S c S o \frac{d}{d r}\left(r \frac{d \theta^{k}}{d r}\right) \\
& E_{N}^{k}=\frac{d}{d r}\left(r \frac{d \omega^{k}}{d r}\right)-\omega A r \frac{d \omega^{k}}{d r}-\left(\frac{1}{r}-\frac{2 \Delta r}{A}\right) \omega^{k}-\frac{\Delta}{A} \frac{d w^{k}}{d r}
\end{aligned}
$$


Where $\mathrm{w}^{\mathrm{k}},, \theta^{\mathrm{k}}, \phi^{\mathrm{k}}$ and $\omega^{\mathrm{k}}$ are values of $\mathrm{w}, \theta$ and $\omega$ in the arbitrary element $\mathrm{e}_{\mathrm{k}}$. These are expressed as linear combinations in terms of respective local nodal values.

$$
\begin{aligned}
& w^{k}=w_{1}^{k} \psi_{1}^{k}+w_{2}^{k} \psi_{2}^{k}+w_{3}^{k} \psi_{3}^{k}=\sum_{i=1}^{3} w_{i}^{k} \psi_{i}^{k} \\
& \theta^{k}=\theta_{1}^{k} \psi_{1}^{k}+\theta_{2}^{k} \psi_{2}^{k}+\theta_{3}^{k} \psi_{3}^{k}=\sum_{i=1}^{3} \theta_{i}^{k} \psi_{i}^{k} \\
& \phi^{k}=\phi_{1}^{k} \psi_{1}^{k}+\phi_{2}^{k} \psi_{2}^{k}+\phi_{3}^{k} \psi_{3}^{k}=\sum_{i=1}^{3} \phi_{i}^{k} \psi_{i}^{k} \\
& \omega^{k}=\omega_{1}^{k} \psi_{1}^{k}+\omega_{2}^{k} \psi_{2}^{k}+\omega_{3}^{k} \psi_{3}^{k}=\sum_{i=1}^{3} \omega_{i}^{k} \psi_{i}^{k}
\end{aligned}
$$

Where $\psi_{1}^{k}, \psi_{2}^{k}, \ldots .$. etc are Lagrange's quadratic polynomials.

Substuting eqn(15) in equations $(11-14)$ and evaluating the resulting integral we get local stiffness matrices. These matrices are assembled into global matrix by using inter element continuity, equilibrium and boundary conditions. These global matrices are solved by iteration procedure. The iteration process is repeated until the convergence is obtained i.e. $\left|u_{i+1}-u_{i}\right|<10^{-6}$.

\section{NUSSELT NUMBER, SHERWOOD NUMBER:}

The rate of heat transfer (Nusselt number) is evaluated using the formula $N u=-\left(\frac{d \theta}{d r}\right)_{r=1,2}$

The rate of mass transfer (Sherwood number) is evaluated using the formula $S h=-\left(\frac{d \phi}{d r}\right)_{r=1,2}$

COMPARISON: In the absence of Heat sources $(\alpha=0)$ the results are in good agreement with those of Prasuna et al[17]

Table - 1

\begin{tabular}{|l|l|l|l|l|l|l|l|l|l|l|l|l|l|}
\hline \multicolumn{4}{|c|}{ Parameters } & \multicolumn{4}{|c|}{ Prasuna et al(28) } & \multicolumn{4}{c|}{ Present results $(\boldsymbol{\alpha}=\mathbf{0})$} \\
\hline $\mathrm{N}$ & $\mathrm{Ec}$ & $\gamma$ & $\Delta$ & $\lambda$ & $\mathrm{Nu}(1)$ & $\mathrm{Nu}(2)$ & $\mathrm{Sh}(1)$ & $\mathrm{Sh}(2)$ & $\mathrm{Nu}(1)$ & $\mathrm{Nu}(2)$ & $\mathrm{Sh}(1)$ & $\mathrm{Sh}(2)$ \\
\hline 1 & 0.01 & 0.5 & 1 & 0.1 & 2.8636 & -5.1236 & 4.2065 & -10.781 & 2.8632 & -5.1237 & 4.2068 & -10.780 \\
\hline 2 & 0.01 & 0.5 & 1 & 0.1 & 6.4175 & -11.548 & 6.7759 & -15.409 & 6.4173 & -11.546 & 6.7756 & -15.408 \\
\hline-0.5 & 0.01 & 0.5 & 1 & 0.1 & -0.8592 & -1.5012 & 1.7038 & -3.6098 & -0.8591 & -1.5011 & 1.7036 & -3.6096 \\
\hline-1.5 & 0.01 & 0.5 & 1 & 0.1 & 0.1787 & -0.01638 & -0.9098 & -1.5997 & 0.1779 & -0.01633 & -0.9099 & -1.5994 \\
\hline 1 & 0.03 & 0.5 & 1 & 0.1 & 1.8355 & -3.3072 & 4.2058 & -10.7794 & 1.8356 & -3.3071 & 4.2055 & -10.7796 \\
\hline 1 & 0.05 & 0.5 & 1 & 0.1 & 2.8756 & -5.1236 & 4.2065 & -10.8802 & 2.8754 & -5.1233 & 4.2066 & -10.9801 \\
\hline 1 & 0.01 & 1.5 & 1 & 0.1 & 2.8853 & -5.1777 & 3.2232 & -8.7041 & 2.8857 & -5.1774 & 3.2229 & -8.7042 \\
\hline 1 & 0.01 & 0.5 & 3 & 0.1 & 1.778 & -5.2273 & 1.5148 & -5.5912 & 1.779 & -5.2271 & 1.5149 & -5.5911 \\
\hline 1 & 0.01 & 0.5 & 5 & 0.1 & 1.035 & -5.0836 & 4.0588 & -8.4699 & 1.033 & -5.0833 & 4.0589 & -8.4696 \\
\hline 1 & 0.01 & 0.5 & 1 & 0.3 & 2.8636 & -2.8052 & 3.0811 & -6.5156 & 2.8633 & -2.8051 & 3.0810 & 2.8636 \\
\hline 1 & 0.01 & 0.5 & 1 & 0.5 & 2.9613 & -5.1236 & 3.4047 & -9.5098 & 2.9611 & -5.1233 & 3.4049 & -9.5099 \\
\hline
\end{tabular}

\section{RESULTS AND DISCUSSION:}

In this analysis we investigate the effect of thermo-diffusion, dissipation and chemical reaction on mixed convective heat and mass transfer flow of a micro polar fluid through a porous medium in circular annulus between the cylinders $r=a$ and $r=b$ which are maintained at constant temperature and concentration. The non-linear coupled equations governing the flow heat and mass transfer are solved by Galerkin finite element analysis with quadratic approximation functions.

Fig. 1 shows the effect of thermo-diffusion effect on w. It can be seen from the graphs that higher the thermo-diffusion effect larger $|w|$.Fig. 2 represents $w$ with chemical reaction parameter $\gamma$. It is found that the magnitude of $\mathrm{w}$ reduces in degenerating generating chemical reaction case and 
enhances in the generating chemical reaction case. Also we find that w exhibits a reversal flow for $\gamma \leq 1.5$ and for $|\gamma|(<0)$. The region of reversal flow enlarges with increase in The variation of w with buoyancy ration $\mathrm{N}$ is shown in Fig. 3 . When the molecular buoyancy force dominates over the thermal force $|w|$ enhances when the buoyancy forces are in the same direction and for the forces acting in opposite direction, $|w|$ depreciates in the flow region. Also we find that $w$ exhibits a reversal flow The effect of dissipation on $\mathrm{w}$ is exhibited in Fig.4. It is found that higher the dissipative effect smaller the magnitude of w. Fig.5 represents w with micropolar parameter $(\Delta)$.It can be seen from the profiles that the axial velocity reduces with increase in $\Delta \leq 1.5$ and for higher $\Delta \geq 2.0$,we notice an enhancement in $\mathrm{w}$ in the entire flow region. Fig. 6 represents $\mathrm{w}$ with suction parameter $\lambda$.It is found that $|w|$ enhances with increase in $\lambda$.An increase in A enhances the magnitude of $\mathrm{W}$ (fig.7).

The non-dimensional temperature $(\theta)$ is exhibited in Figs. 8-14 for different parametric values. Higher the thermo-diffusion effects smaller the actual temperature in the flow region(fig.8).Fig. 9 represents $\theta$ with chemical reaction parameter $\gamma$. It is found that the actual temperature reduces with $\gamma$ in both degenerating and generating cases. Fig. 10 represents $\theta$ with buoyancy ratio $\mathrm{N}$. It can be seen from the profiles that the molecular buoyancy force dominates the actual temperature reduces in the flow region, when the buoyancy forces are in the same direction and for the forces acting in opposite direction, it enhances in the flow region. The variation of $\theta$ with Eckert number Ec is shown in the Fig. 11. It is found that the actual temperature reduces with Ec in the entire flow region.Fig. 12 shows the variation $\theta$ with micropolar parameter $\Delta$.Higher the values of $\Delta$,lesser the actual temperature in the flow region. The variation of $\theta$ with $\lambda$ is exhibited in Fig.13. It is found that the actual temperature experiences an enhancement with increase in suction parameter.Fig.14 exhibits $\theta$ with micropolar parameter A.It can be seen from the graphs that the actual temperature enhances with increase in A.

The concentration distribution (C) is exhibited in Figs.15-21. Fig.15 shows the variation of $\mathrm{C}$ with Soret parameter So.Higher the thermo-diffusion effects larger the actual concentration in the flow region.From fig. 16 we find that the actual concentration experience a depreciation in both the degenerating and generating chemical reaction cases. The variation of $\mathrm{C}$ with buoyancy ratio(N) is shown in fig.17.It can be seen from the profiles that when the molecular buoyancy force dominates over the thermal buoyancy force the actual concentration reduces irrespective of the directions of the buoyancy forces. The actual concentration reduces with increase in micropolar parameters, $\Delta$ (fig.19) and A(fig.21). The variation of $\theta$ with $\lambda$ is exhibited in Fig.20 shows the variation of $\mathrm{C}$ with suction parameter $\lambda$. It is found that the actual temperature experiences an enhancement with increase in suction parameter.

The micro rotation $(\omega)$ is shown in Figs. 22-28 for different parametric values. Fig.23 represents $\omega$ with chemical reaction parameter $\gamma$. It is found that the micro rotation the magnitude of $\omega$ enhances in both the degenerating and generating cases. Higher the thermo-diffusion effects larger the magnitude of the angular velocity(fig.22). Fig. 24 represents with $\omega$ buoyancy ratio N. It is found that the magnitude of $\omega$ increases with $|\mathrm{N}|$ irrespective of the directions of the buoyancy forces. Higher the dissipation lesser $|\omega|$ in the entire flow region. An increase in micropolar parameter $\Delta$ reduces $|\omega|$ in the region(1.1,1.5) and increases in the region(1.6,1.9)(fig.26).An increase in suction parameter $\lambda$ enhances $|\omega|$ in the flow region(fig.27).From fig.28 we find that $|\omega|$ enhances wit increase in micropolar parameter A.

The rate of heat transfer (Nusselt number) $(\mathrm{Nu})$ at $\mathrm{r}=1$ and $\mathrm{r}=2$ is shown in Tables 2 for different parametric values. $|\mathrm{Nu}|$ enhances at $\mathrm{r}=1$ and reduces at $\mathrm{r}=2$ with increase in $|\mathrm{N}|$. An increase in suction parameter $\lambda$ reduces $|\mathrm{Nu}|$ at the outer cylinder at $r=1$. The variation of $\mathrm{Nu}$ with micro rotation parameter $\mathrm{A}$ and viscosity ratio parameter $\Delta$ shows that $|\mathrm{Nu}|$ enhances with $\mathrm{A}$ and depreciates with $\Delta$ at $\mathrm{r}=1$ and 2 . The variation of $\mathrm{Nu}$ with $\mathrm{Ec}$ shows that higher the dissipative heat 
smaller $|\mathrm{Nu}|$.Also $|\mathrm{Nu}|$ experiences and enhances at $\mathrm{r}=1$ and 2, with increase in the chemical reaction parameter $\gamma$.

The rate of mass transfer (Sherwood number) at $r=1$ and 2 is exhibited in Tables 2 for different parametric values. The variation of Sh with buoyancy ratio $\mathrm{N}$ shows that when the molecular buoyancy force dominates over the thermal buoyancy force $|\mathrm{Sh}|$ enhances at $\mathrm{r}=1$ and reduces at $\mathrm{r}=2$ when the buoyancy forces are in the same direction and for the forces acting in opposite direction it depreciates at $\mathrm{r}=1$ and increases at $\mathrm{r}=2$. We find that the rate of mass transfer enhances with $\lambda$ and reduces with $\mathrm{Ec}$ at $\mathrm{r}=1$ and 2.An increase in $\Delta$ reduces at $\mathrm{r}=1$ and enhances at $\mathrm{r}=2$ while it enhance4s with $\mathrm{A}$ at both cylinders. Higher the thermo-diffusivity smaller $|\mathrm{Sh}|$ at both the cylinders. With respect to $\gamma$ we find that $|\mathrm{Sh}|$ reduces at both the cylinders in degenerating and generating chemical reaction cases.

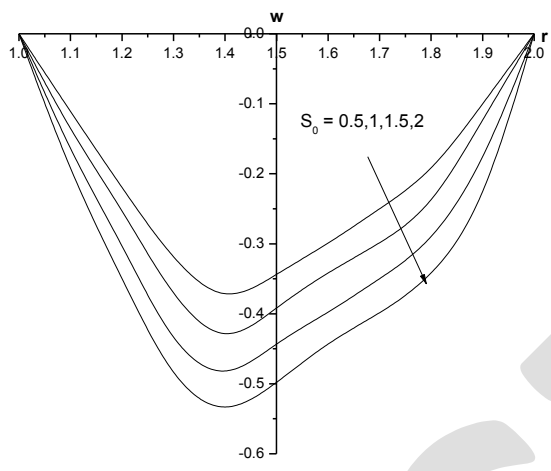

Fig.1 Variation w with $\mathrm{S}_{0}$ $\gamma=0.5, \mathrm{~N}=1, \Delta=0.5, \lambda=0.3, \mathrm{~A}=0.5$

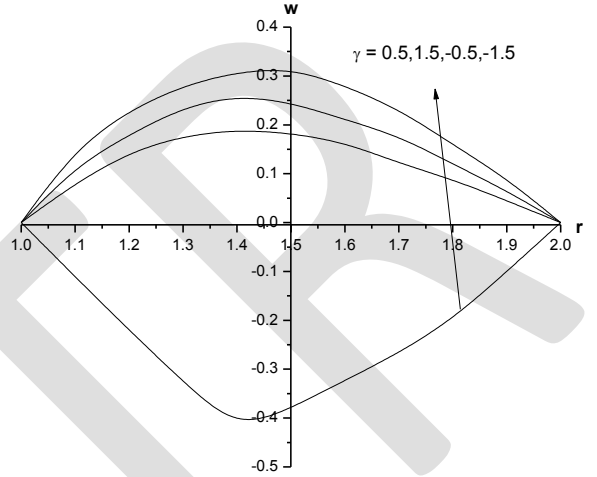

Fig.2: Variation of $w$ with $\gamma$ $\mathrm{S}_{0}=0.5, \mathrm{~N}=1, \Delta=0.5, \lambda=0.3, \mathrm{~A}=0.5$

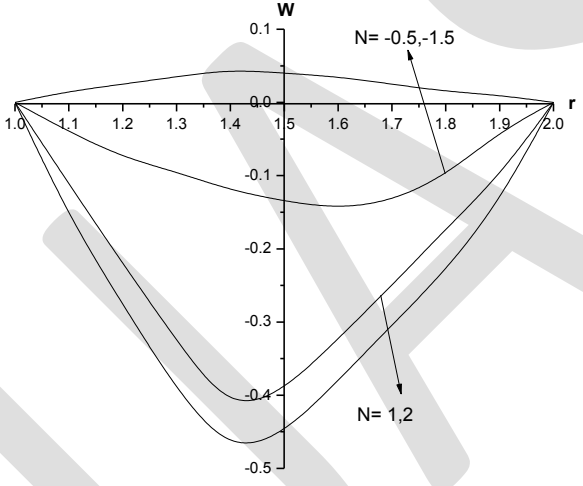

Fig. 3 Variation w with $\mathrm{N}$ $\mathrm{S}_{0}=0.5, \gamma=0.5, \Delta=0.5, \lambda=0.3, \mathrm{~A}=0.5$

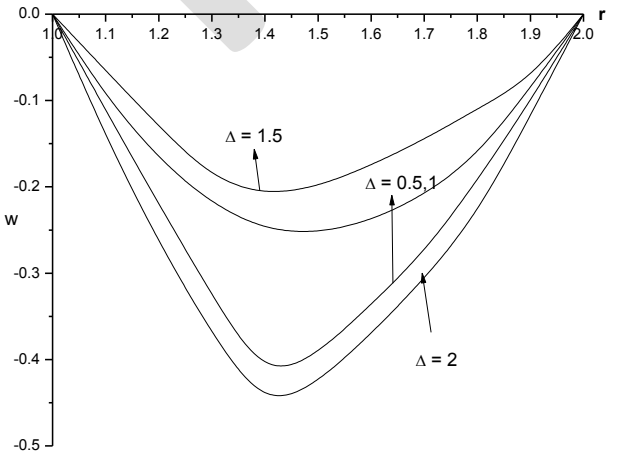

Fig.5 Variation w with $\Delta$ $\mathrm{S}_{0}=0.5, \gamma=0.5, \mathrm{~N}=1, \lambda=0.3, \mathrm{~A}=0.5$

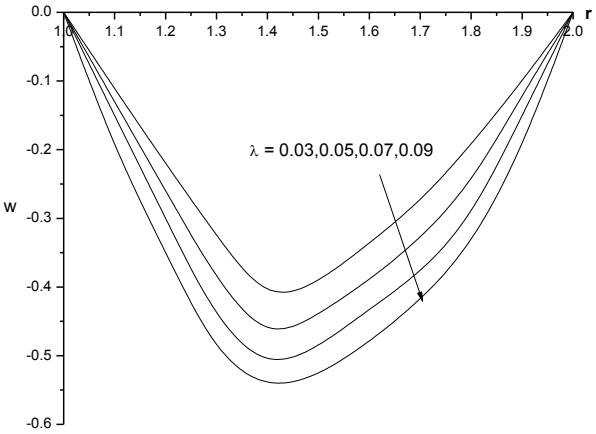

Fig.4: Variation of $w$ with $\lambda$

$\mathrm{S}_{0}=0.5, \gamma=0.5, \mathrm{~N}=1, \Delta=0.5, \mathrm{~A}=0.5$
Fig.4: Variation of w with Ec $\mathrm{S}_{0}=0.5, \gamma=0.5, \mathrm{~N}=1, \Delta=0.5, \lambda=0.3, \mathrm{~A}=0.5$ 


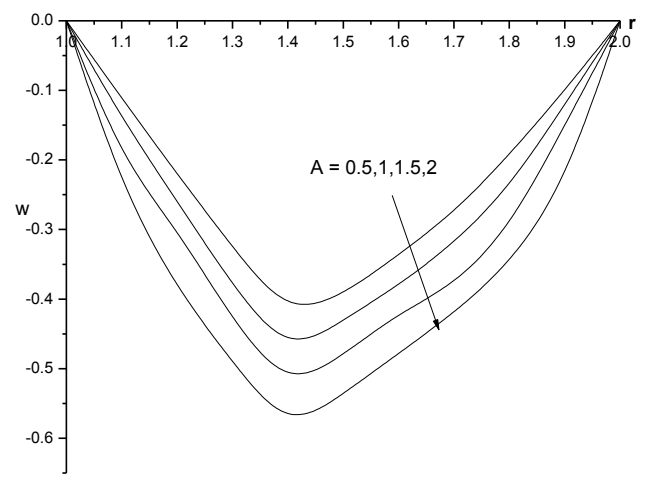

Fig.7 Variation w with A $\mathrm{S}_{0}=0.5, \gamma=0.5, \mathrm{~N}=1, \Delta=0.5, \lambda=0.3$

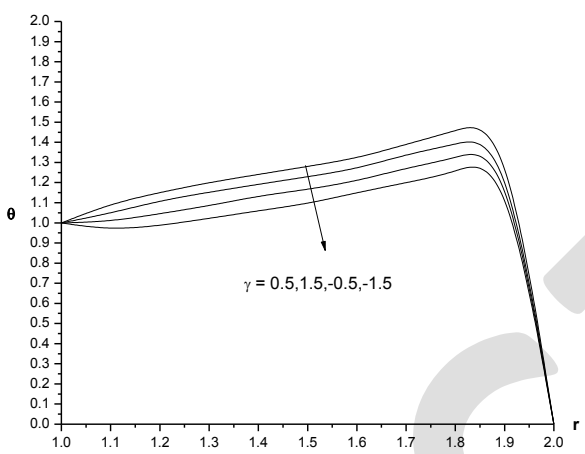

Fig.9 Variation $\theta$ with $\gamma$

$\mathrm{S}_{0}=0.5, \mathrm{~N}=1, \Delta=0.5, \lambda=0.3, \mathrm{~A}=0.5$

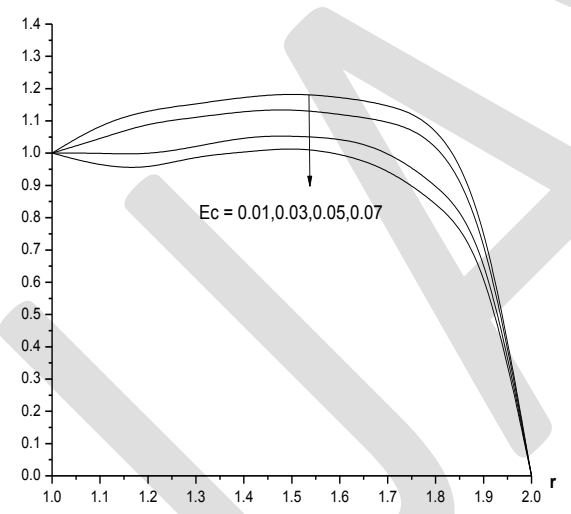

Fig.11 Variation $\theta$ with Ec

$\mathrm{S}_{0}=0.5, \gamma=0.5, \mathrm{~N}=1, \Delta=0.5, \lambda=0.3, \mathrm{~A}=0.5$

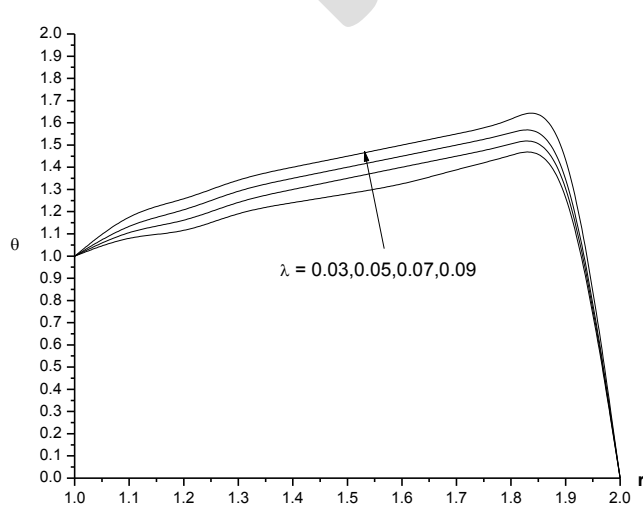

Fig.13 Variation $\theta$ with $\lambda$

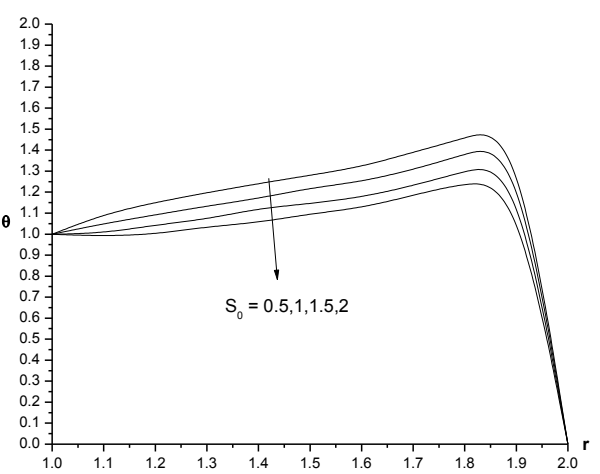

Fig.8: Variation of $\theta$ with $\mathrm{S}_{0}$ $\gamma=0.5, \mathrm{~N}=1, \Delta=0.5, \lambda=0.3, \mathrm{~A}=0.5$

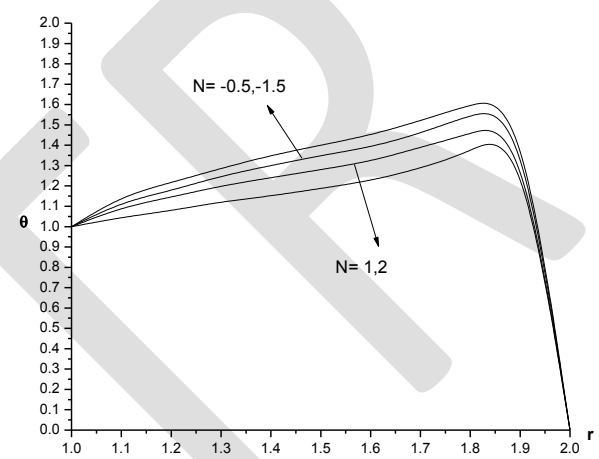

Fig.10: Variation of $\theta$ with $\mathrm{N}$

$\mathrm{S}_{0}=0.5, \gamma=0.5, \Delta=0.5, \lambda=0.3, \mathrm{~A}=0.5$

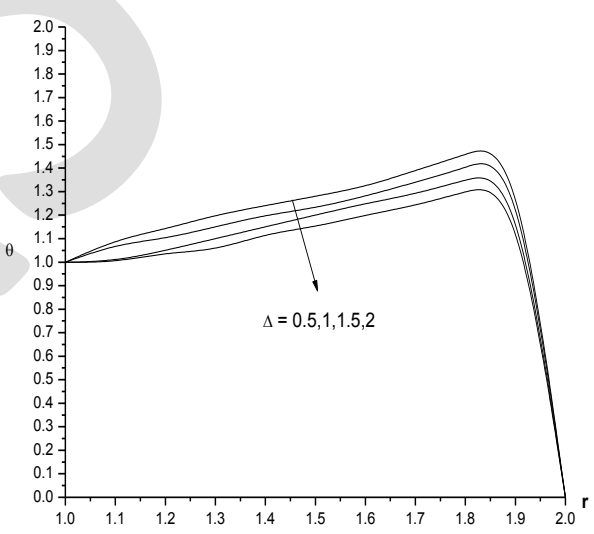

Fig.12: Variation of $\theta$ with $\Delta$

$\mathrm{S}_{0}=0.5, \gamma=0.5, \mathrm{~N}=1, \lambda=0.3, \mathrm{~A}=0.5$

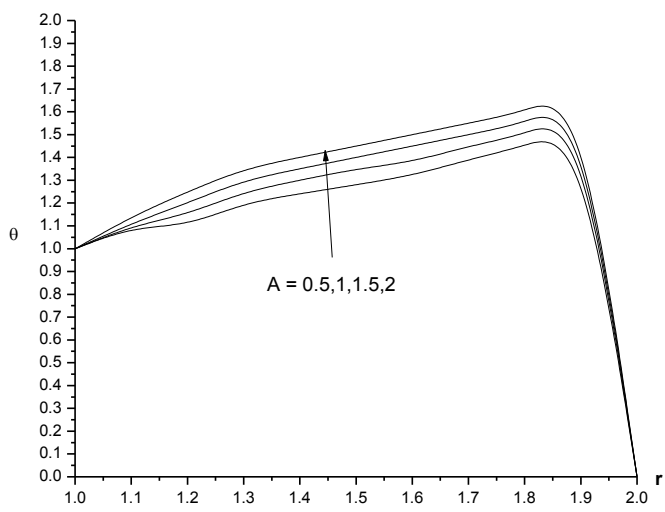

Fig.14: Variation of $\theta$ with A 
DOI : https://dx.doi.org/10.26808/rs.st.i8v1.12

International Journal of Advanced Scientific and Technical Research

Issue8 volume 1 January-February 2018 Available online on http://www.rspublication.com/ijst/index.html

$\mathrm{S}_{0}=0.5, \gamma=0.5, \mathrm{~N}=1, \Delta=0.5, \mathrm{~A}=0.5$

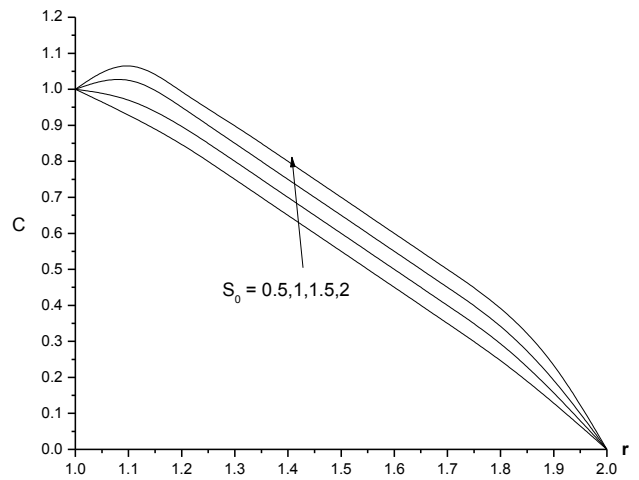

Fig.15 Variation $\mathrm{C}$ with $\mathrm{S}_{0}$

$\gamma=0.5, \mathrm{~N}=1, \Delta=0.5, \lambda=0.3, \mathrm{~A}=0.5$

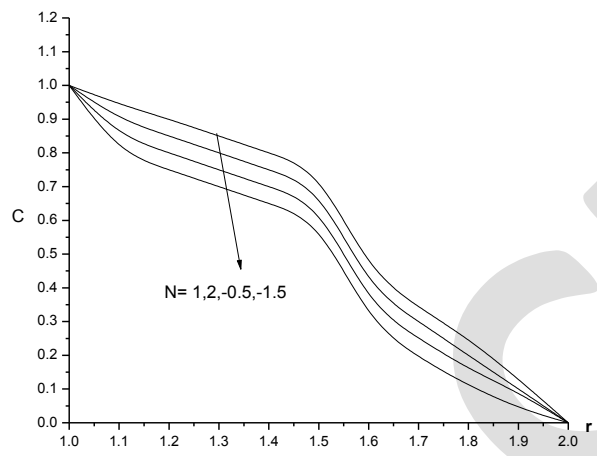

Fig. 17 Variation $\mathrm{C}$ with $\mathrm{N}$

$\mathrm{S}_{0}=0.5, \gamma=0.5, \Delta=0.5, \lambda=0.3, \mathrm{~A}=0.5$

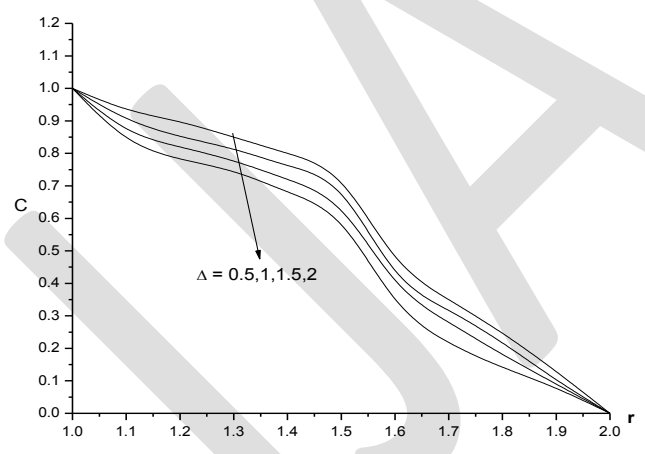

Fig.19 Variation $\mathrm{C}$ with $\Delta$

$\mathrm{S}_{0}=0.5, \gamma=0.5, \mathrm{~N}=1, \lambda=0.3, \mathrm{~A}=0.5$

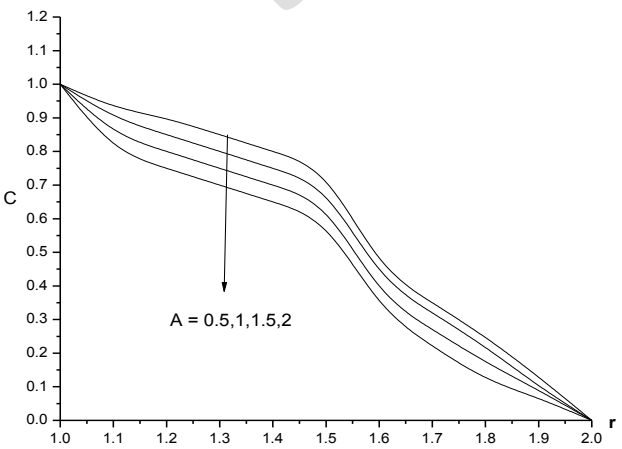

Fig.21 Variation $\mathrm{C}$ with $\mathrm{A}$

$\mathrm{S}_{0}=0.5, \gamma=0.5, \mathrm{~N}=1, \Delta=0.5, \lambda=0.3$
$\mathrm{S}_{0}=0.5, \gamma=0.5, \mathrm{~N}=1, \Delta=0.5, \lambda=0.3$

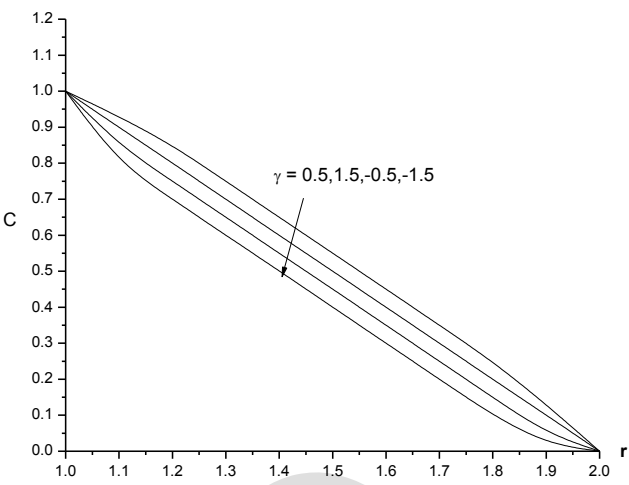

Fig.16: Variation of $\mathrm{C}$ with $\gamma$ $\gamma=0.5, \mathrm{~N}=1, \Delta=0.5, \lambda=0.3, \mathrm{~A}=0.5$

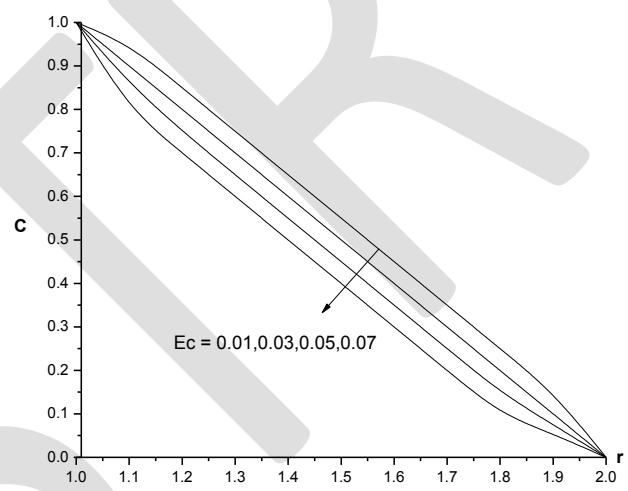

Fig.18: Variation of $\mathrm{C}$ with $\mathrm{Ec}$ $\mathrm{S}_{0}=0.5, \gamma=0.5, \mathrm{~N}=1, \Delta=0.5, \lambda=0.3, \mathrm{~A}=0.5$

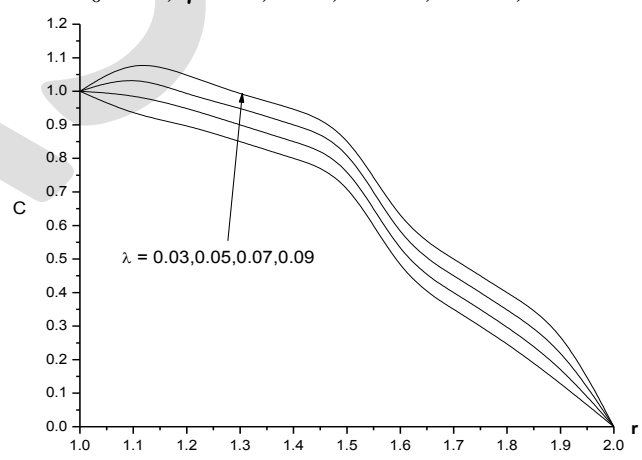

Fig.20: Variation of $\mathrm{C}$ with $\lambda$ $\mathrm{S}_{0}=0.5, \gamma=0.5, \mathrm{~N}=1, \Delta=0.5, \mathrm{~A}=0.5$

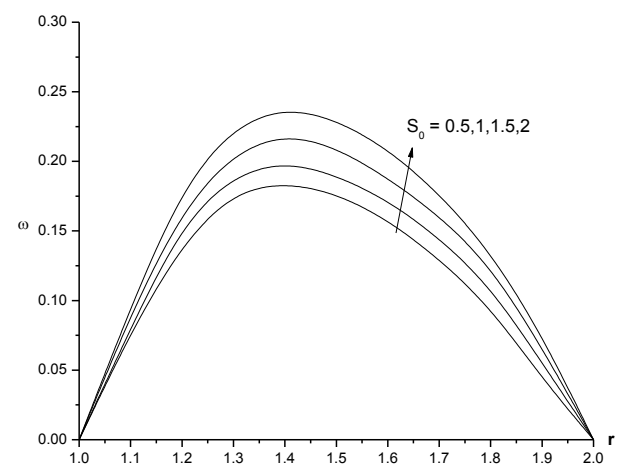

Fig.22: Variation of $\omega$ with $\mathrm{S}_{0}$ $\gamma=0.5, \mathrm{~N}=1, \Delta=0.5, \lambda=0.3, \mathrm{~A}=0.5$ 


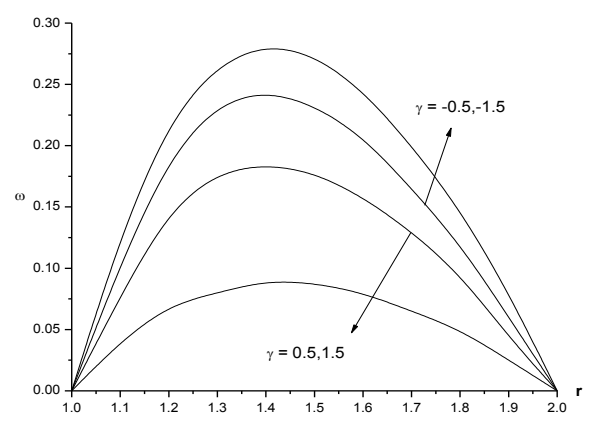

Fig.23 Variation $\omega$ with $\gamma$ $\mathrm{S}_{0}=0.5, \mathrm{~N}=1, \Delta=0.5, \lambda=0.3, \mathrm{~A}=0.5$

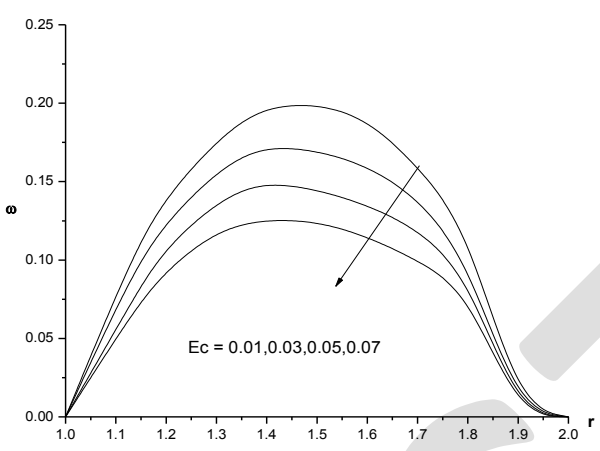

Fig. 25 Variation $\omega$ with Ec $\mathrm{S}_{0}=0.5, \gamma=0.5, \mathrm{~N}=1, \Delta=0.5, \lambda=0.3, \mathrm{~A}=0.5$

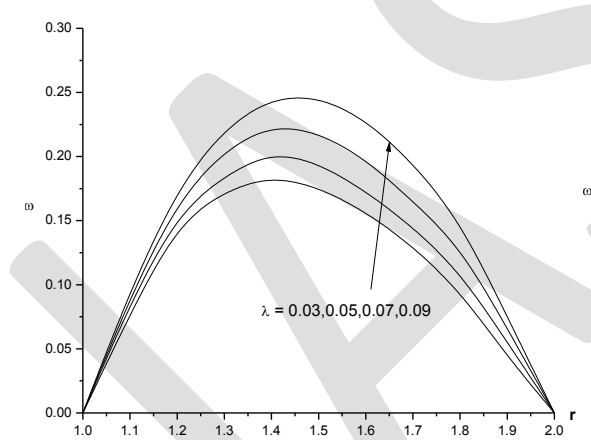

Fig. 27 Variation $\omega$ with $\lambda$

$\mathrm{S}_{0}=0.5, \gamma=0.5, \mathrm{~N}=1, \Delta=0.5, \mathrm{~A}=0.5$

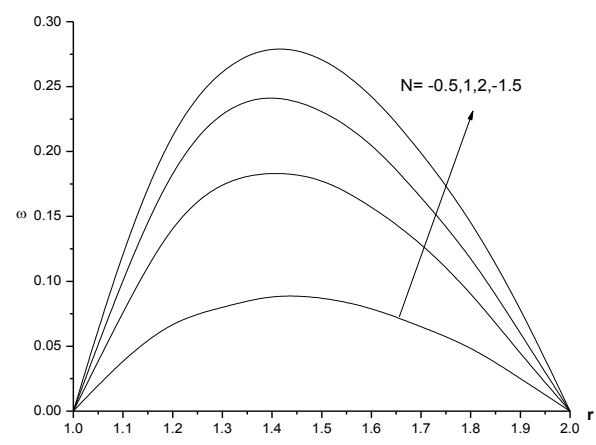

Fig.24: Variation of $\omega$ with $\mathrm{N}$ $\mathrm{S}_{0}=0.5, \gamma=0.5, \Delta=0.5, \lambda=0.3, \mathrm{~A}=0.5$

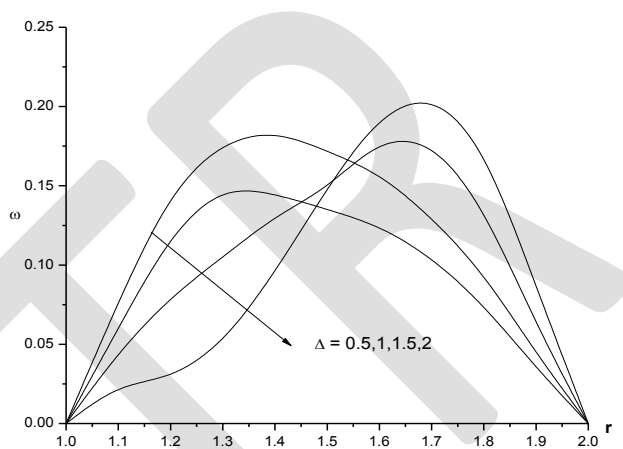

Fig.26: Variation of $\omega$ with $\Delta$ $\mathrm{S}_{0}=0.5, \gamma=0.5, \mathrm{~N}=1, \lambda=0.3, \mathrm{~A}=0.5$

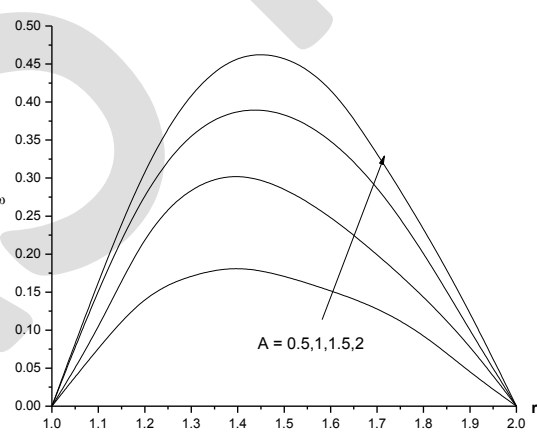

Fig.28: Variation of $\omega$ with A $\mathrm{S}_{0}=0.5, \gamma=0.5, \mathrm{~N}=1, \Delta=0.5, \lambda=0.3$

Table -2 : Nusselt Number(Nu), Sherwood Number (Sh)

\begin{tabular}{|l|l|l|l|l|l|l|c|c|c|c|}
\hline $\mathbf{N}$ & $\mathbf{S o}$ & $\gamma$ & $\mathbf{E c}$ & $\Delta$ & $\lambda$ & $\mathrm{A}$ & $\mathbf{N u}(\mathbf{1})$ & $\mathbf{N u}(\mathbf{2})$ & $\mathbf{S h}(\mathbf{1})$ & $\mathbf{S h}(\mathbf{2})$ \\
\hline 1 & 0.5 & 0.5 & 0.01 & 2 & 0.2 & 0.5 & 27.5626 & -39.4221 & 23.8952 & -2.2822 \\
\hline 2 & 0.5 & 0.5 & 0.01 & 2 & 0.2 & 0.5 & 27.5632 & -39.4198 & 23.8968 & -2.28153 \\
\hline-0.5 & 0.5 & 0.5 & 0.01 & 2 & 0.2 & 0.5 & 27.5617 & -39.4252 & 23.8922 & -2.28306 \\
\hline-1.5 & 0.5 & 0.5 & 0.01 & 2 & 0.2 & 0.5 & 27.5614 & -39.4261 & 23.8912 & -2.28329 \\
\hline 1 & 1.0 & 0.5 & 0.01 & 2 & 0.2 & 0.5 & 27.5939 & -39.7369 & 24.0929 & -2.49708 \\
\hline 1 & 1.5 & 0.5 & 0.01 & 2 & 0.2 & 0.5 & 27.6093 & -39.9767 & 24.3374 & -2.77969 \\
\hline 1 & 0.5 & 1.5 & 0.10 & 2 & 0.2 & 0.5 & 27.5545 & -39.3852 & 23.8942 & -2.28126 \\
\hline 1 & 0.5 & -0.5 & 0.01 & 2 & 0.2 & 0.5 & 27.5341 & -39.2921 & 23.8921 & -2.27886 \\
\hline 1 & 0.5 & -1.5 & 0.01 & 2 & 0.2 & 0.5 & 27.4928 & -39.1051 & 23.8878 & -2.27405 \\
\hline 1 & 0.5 & 0.5 & 0.03 & 2 & 0.2 & 0.5 & 27.5078 & -39.4056 & 23.9456 & -2.30456 \\
\hline 1 & 0.5 & 0.5 & 0.05 & 2 & 0.2 & 0.5 & 27.5989 & -40.0234 & 23.9987 & -2.33456 \\
\hline 1 & 0.5 & 0.5 & 0.01 & 4 & 0.2 & 0.5 & 27.1435 & -34.4998 & 23.8866 & -2.30144 \\
\hline 1 & 0.5 & 0.5 & 0.01 & 6 & 0.2 & 0.5 & 27.1567 & -34.5078 & 23.8076 & -2.31245 \\
\hline 1 & 0.5 & 0.5 & 0.01 & 2 & 0.4 & 0.5 & 27.5879 & -39.4567 & 23.9966 & -2.28998 \\
\hline 1 & 0.5 & 0.5 & 0.01 & 2 & 0.6 & 0.5 & 27.6089 & -39.4998 & 24.0345 & -2.29134 \\
\hline 1 & 0.5 & 0.5 & 0.01 & 2 & 0.2 & 1.0 & 28.0345 & -39.4689 & 24.0134 & -2.30234 \\
\hline 1 & 0.5 & 0.5 & 0.01 & 2 & 0.2 & 1.5 & 28.1234 & -39.9996 & 24.5678 & -2.32456 \\
\hline
\end{tabular}




\section{CONCLUSIONS:}

$>$ The velocity reduces in the degenerating chemical reaction case and enhances in the generating case, the temperature and concentration reduces and the micro rotation enhances in both generating and degenerating chemical reaction cases.

$>$ An increasing suction parameter $\lambda$ enhances $w, \theta, \mathrm{C}$ and the angular velocity $\omega$.

$>$ The velocity and microrotation enhances and temperature ,Concentration reduces with increasing $\mathrm{N}>0$ and while for $\mathrm{N}<0$, the axial velocity, Concentration reduces, the the temperature and microrotation enhances in the flow region.

$>$ An increasing Eckert number Ec reduces the velocity, temperature, concentration and microrotation.

$>$ Higher the thermo-diffusion effect larger the velocity, concentration and microrotation while smaller the temperature in the flow region.

$>|\tau|$, reduces and $|\mathrm{Nu}| \&|\mathrm{Sh}|$ enhances at $\mathrm{r}=1$ and $\mathrm{r}=2$ with increase in Soret parameter So.

$>$ An increasing the micropolar parameter $\lambda$ enhances $|\mathrm{Nu}|,|\mathrm{Sh}|$ at both the cylinders.

$>$ Higher the dissipative heat smaller $|\mathrm{Sh}|$ and $|\mathrm{Nu}|$ at $\mathrm{r}=1$ and 2

\section{REFERENCES:}

1. Agarwal R.S. and Dhanpal C.: Numerical solution of micropolar fluid flow and heat transfer between two co-axial porous circular cylinders. Int. J. Eng. Sci., 26(11) pp. 1133-1142 (1988).

2. Barletta A.: Int. J. Heat Mass Transfer, Vol. 40, pp. 15-26 (1997).

3. Eringen A.C.: The theory of micropolar fluids - theory and application. Birkhauser, Bostan (1966).

4. Fand R.M. and Brucker J.: Int. J. Heat and Mass Transfer. p. 723 (1986).

5. GiampietroI Fabbri: Int. J. Heat and Mass Transfer, p. 3003 (2004).

6. Gebhart B. and Mollendorf J.: Fluid Mech., Vol. 38, pp. 107 (1969).

7. Lukaszewicz G.: Micropolar fluids, theory and applications, Birkhauser, Bostan (1999).

8. Murthy D.V.S. and Singh P.: Int. J. Heat and Mass Transfer, (1997).

9. Murthy J.V.R. and Bahali N.K.: Steady flow of micropolar fluid through a circular pipe under a transverse magnetic field with constant suction/injection, (2009).

10. Nakayama A. and Pop I.: Int. Communications in Heat and Mass Transfer, (1989).

11. Panja S., Sengupta P.R. and Debnath L.: Hydro magnetic flow of Reiner - Rivlin fluid between two coaxial circular in cylinders with porous walls. Computers Math. Applic. 32 (2). pp. 1-4, (1986).

12. Shivashankar I.S., Prasanna B.M.R., Sankar M. and Sreedhara S.: Numerical study of natural convection in vertical porous Annuli in the presence of magnetic field, (2009).

13. Soundalgekar V.M. and Jaisawal B.S.D., Uplekar A.G. and Takhar H.S.: Appl. Mech and Engg, Vol. 4, pp. 203-218 (1999).

14. Sreevani M.: Mixed convective heat and mass transfer through a porous medium in channels with dissipative effects Ph.D. Thesis, S.K. University, Anantapur (A.P.), (2003).

15. Soundalgekar V.M. and Pop I.: Int. J. Heat Mass Transfer, Vol. 17, pp. 85-90 (1974).

16. Verma P.D.S. and Singh M.: Magnetic fluid flow through porous annulus. Int. J. Non-linear Mech., 16(314), pp. 371-37 (1981).

17. Sulochana C and K. Gnana Prasunamba, MHD Double diffusive convective heat transfer flow of micro polar fluid in cylindrical annulus., presented in International Conference in Mathematics, V.S.K. University, Ballary, (2014). 